\title{
Phase-Space Invariants as Indicators of the Critical Behavior of Nanoaggregates
}

\author{
Vincenzo Aquilanti, ${ }^{1}$ Andrea Lombardi, ${ }^{1}$ Mikhail B. Sevryuk,,${ }^{1, *}$ and Ersin Yurtsever ${ }^{2}$ \\ ${ }^{1}$ Dipartimento di Chimica, Università di Perugia, I-06123 Perugia, Italy \\ ${ }^{2}$ College of Arts and Sciences, Koç University Rumelifeneri yolu, Sar1yer Istanbul, Turkey 34450 \\ (Received 27 November 2003; published 9 September 2004; publisher error corrected 10 September 2004) \\ A phase-space approach is proposed for molecular dynamics simulations, which serve as a bridge \\ between detailed descriptions of microscopic world and macroscopic properties of matter. The \\ introduction-aside from the angular momentum of spatial rotations — of other "hyperangular" \\ momenta (the overall grand angular momentum of a cluster of particles and those describing the \\ deformation and rearrangement modes) permits one to analyze different degrees of freedom and to \\ extract, from simulation data, a kinetic energy partition in terms of phase-space invariants. Model \\ calculations illustrate how these provide specific signatures of critical behavior, such as energy \\ thresholds for openings of chaotic pathways in small clusters and for phase transitions in \\ nanoaggregates.
}

DOI: 10.1103/PhysRevLett.93.113402

PACS numbers: 36.40.Sx, 36.40.Ei, 45.50.Jf

The dynamics of aggregates constituted by a large number of particles is currently simulated by often extensive computer generated trajectories based on classical mechanics, quantum mechanical treatments being not likely to be extendable to systems containing more than a few particles. An ample phenomenology is being accumulated regarding signatures of chaotic versus regular behavior [1-4] and manifestations of phenomena which in small aggregates are seen as the nano- or meso-scale analogues of the phase transitions occurring in macroscopic systems [5-9]. To assist in the understanding and use of the voluminous body of data coming out of such classical molecular dynamics simulations, here we propose to exploit some progress essentially inspired by developments in the (exact) quantum mechanical approach to few-body problems. Besides serving for pinpointing the above mentioned phenomena and for contributing as interpretation tools of molecular dynamics simulations, the analysis is also of perspective value for the formulation of approximate quantum treatments.

The quantum mechanical approach relies upon coordinate representations based on moments of inertia and deformation parameters and suggests a search for invariant quantities [10-12]; their classical counterparts have been recently illustrated following individual classical trajectories of small cluster dynamics [13]. The further step, which we take here, involves moving towards global properties of an ensemble of particles and studying features, such as specific heats and phase transitions [5-8], which require long-time averages over batches of trajectories. Our examples will consider microcanonical systems [8], as appropriate for nanoaggregates. Most of previous work focused on invariants in the configuration space, but since classical simulations provide instantaneous full phase-space information (typically the Cartesian coordinates and momenta of individual particles), our aim is to extract phase-space invariants in order to compact the available information. We will show how the concept of the so called hyperangular momenta (phase-space quantities generalizing the grand angular momentum introduced by Smith for a system of particles [14]) can lead to the definition of other invariants, associated to the deformation and rearrangement modes, which can be straightforwardly computed by quantities available in any current molecular dynamics simulation software. This approach allows us to present a partition of the kinetic energy into terms including not only those due to the rotation of the system as a whole, and those due to size and shape variations (the overall breathing and deformation contributions, respectively) [11,12], but also those arising out of particle interchanges (the kinematic rotations [10-12,15-23]). Two examples, for three and 13 particles bound by Lennard-Jones binary forces (simulating well studied clusters such as $\mathrm{Ar}_{3}[1-3]$ and $\mathrm{Ar}_{13}[5,6]$ ), will be presented to demonstrate the relative role of various terms: in particular, the deformation and rearrangement contributions are expected to provide characteristic signatures in correspondence of onsets of chaotic behavior and of phase transitions.

According to the hyperspherical view of classical dynamics, initiated in Ref. [13], the motion of an $N$-body system is described in a $3 \times(N-1)$ hyperspace as that of a vector of variable length $\rho$ (the hyperradius, a quantity measuring the total inertia $I=2 M \rho^{2}$, where $M$ is the total mass of the system and $I$ is the sum of its three principal moments of inertia $I_{1}, I_{2}, I_{3}$ ). The total kinetic energy $T$ in the center-of-mass frame is then expressed as the sum of two terms [14]

$$
T=T_{\rho}+T_{\Lambda},
$$

where

$$
T_{\rho}=\frac{P_{\rho}^{2}}{2 M}, \quad T_{\Lambda}=\frac{\Lambda^{2}}{2 M \rho^{2}} .
$$


Here, $P_{\rho}=M \dot{\rho}=M d \rho / d t$ is the linear momentum conjugated to the hyperradius [14], so the first term accounts for the overall breathing of the system. The second term is associated to the grand angular momentum $\Lambda$, the modulus of the $(3 N-3) \times(3 N-3)$ skew-symmetric tensor $\boldsymbol{\Lambda}$ [14] containing angular momentumlike elements which depend on readily available quantities (the instantaneous coordinates and velocities of the particles). The square of $\Lambda$ is the sum of the squares of all the elements $\Lambda_{m}$ of this tensor:

$$
\Lambda^{2}=\sum_{m=1}^{(3 N-3)(3 N-4) / 2} \Lambda_{m}^{2} .
$$

Moreover, the terms $\Lambda_{m}$ can be partitioned into physically interesting groups in order to obtain the corresponding partition of the $T_{\Lambda}$ component [see Eq. (1)] of the total kinetic energy $T$. From this perspective, the hyperspherical view of classical mechanics allows us to look at the dynamics of a system as at the sharing of the kinetic energy among different kinds of contributing motions that are closely related to the representation given by the symmetric hyperspherical coordinates, widely used in quantum mechanics $[22,23]$.

The instantaneous particle coordinates and velocities are ingredients of $\Lambda$ in very much the same way as of the ordinary (spatial) angular momentum, for which $\Lambda$ is a generalization. Indeed, in the three-dimensional physical space we have the modulus $J$ of the conventional angular momentum vector $\mathbf{J}$ :

$$
J^{2}=J_{x}^{2}+J_{y}^{2}+J_{z}^{2}
$$

where $J_{i}(i=x, y, z)$ are the three components of $\mathbf{J}$. Each component $J_{i}$ is obtained as the sum of $N$ terms that are components of the single particle angular momenta along the $i$-axis. These $N$ terms are the contributions to $J_{i}$ from the individual particles. The quantity $J$ is a constant of the motion, and the property for $J$ of being a phase-space invariant under both spatial (external) rotations and kinematic rotations (particle rearrangements) is obvious. The kinematic (or internal) rotations are rotations in an abstract $(N-1)$-dimensional hyperspace (the so called kinematic space) and deal with those internal motions of particles which leave unaltered $I_{1}, I_{2}$, and $I_{3}$. We therefore construct the kinematic angular vector $\mathbf{K}$ dual to $\mathbf{J}$ such that the square of its modulus $K$ is

$$
K^{2}=\sum_{n=1}^{(N-1)(N-2) / 2} K_{n}^{2},
$$

where $K_{n}$ are the components of $\mathbf{K}$ in the kinematic space. Each component $K_{n}$ is the sum of three terms measuring the contributions to $K_{n}$ coming from the three Cartesian axes in the physical space. One can prove that the quantity $K$ (to be called the kinematic angular momentum) is also a phase-space invariant under both spatial rotations and kinematic rotations. Finally, it is possible to define the angular momentum $L_{\xi}$ associated with the deformation modes of the motion of the particles, i.e., with the variations of the principal moments of inertia $I_{1}, I_{2}, I_{3}$. To this end, it is suitable to introduce the singular values $\xi_{1}, \xi_{2}$, $\xi_{3}$ of the system via the formulas [10-13,21]

$$
\begin{gathered}
\xi_{1}^{2}=\frac{I_{2}+I_{3}-I_{1}}{2 M}, \quad \xi_{2}^{2}=\frac{I_{1}+I_{3}-I_{2}}{2 M}, \\
\xi_{3}^{2}=\frac{I_{1}+I_{2}-I_{3}}{2 M} .
\end{gathered}
$$

They are tied to the global properties of the $N$-body system, which are invariant under kinematic rotations: its content $\xi_{1}^{2} \xi_{2}^{2} \xi_{3}^{2}$ (the volume in an $(N-1)$ hyperspace), its surface area $\xi_{1}^{2} \xi_{2}^{2}+\xi_{1}^{2} \xi_{3}^{2}+\xi_{2}^{2} \xi_{3}^{2}$, and its perimeter $\xi_{1}^{2}+\xi_{2}^{2}+\xi_{3}^{2}$ (the latter is seen to be $\rho^{2}$ ). The variation in shape is described by the phase-space invariant $L_{\xi}$ (the modulus of the vector $\mathbf{L}_{\xi}$ in an abstract three-dimensional space of the triples of singular values) which is proposed to be determined from the relation

$$
\begin{aligned}
\left(L_{\xi} / M\right)^{2}= & \left(\xi_{1} \dot{\xi}_{2}-\xi_{2} \dot{\xi}_{1}\right)^{2}+\left(\xi_{1} \dot{\xi}_{3}-\xi_{3} \dot{\xi}_{1}\right)^{2}+\left(\xi_{2} \dot{\xi}_{3}\right. \\
& \left.-\xi_{3} \dot{\xi}_{2}\right)^{2} .
\end{aligned}
$$

The momenta $J, K$, and $L_{\xi}$ allow us to define further quantities playing the physical role of kinetic energy terms:

$$
T_{J}=\frac{J^{2}}{2 M \rho^{2}}, \quad T_{K}=\frac{K^{2}}{2 M \rho^{2}}, \quad T_{\xi}=\frac{L_{\xi}^{2}}{2 M \rho^{2}} .
$$

The terms $T_{J}, T_{K}$, and $T_{\xi}$ are instantaneous invariants in the phase space [like $T_{\rho}$ and $T_{\Lambda}$ given by Eq. (1)]. Just as $T_{J}$ accounts for contribution to the kinetic energy from the physical angular momentum, $T_{K}$ and $T_{\xi}$ play the same role regarding kinematic rotations and shape deformations. The suggested partition of $T_{\Lambda}$ is [24]:

$$
T_{\Lambda}=T_{J}+T_{K}+T_{\xi}+T_{\mathrm{ac}},
$$

which defines through Eq. (1) and Eq. (2) another instantaneous invariant, the angular coupling energy $T_{\mathrm{ac}}$ : it accounts for non separability and contains, besides the familiar Coriolis-type couplings between spatial rotations and deformation modes, also the corresponding one between the latter and kinematic rotations [10].

This well-defined separability of degrees of freedom is proposed as a physically motivated alternative for aggregates to the usual (vibro-rotational) one of the molecular modes, which leads, for example, to the application of the principle of the equipartition of energy in the theory of heat capacities. Other schemes are being introduced and classified according to the given characteristics of the system in question (a rigorous analysis can be performed of these additional schemes [10]). We will apply such a separation of energy modes to three-atom and 13-atom systems with atoms interacting by a Lennard-Jones $(12,6)$ 
pairwise potential $[1-7,13]$. These are thought to be reasonable models for rare gas clusters such as $\mathrm{Ar}_{3}$ [1-3] and $\mathrm{Ar}_{13}[5,6]$. The data in Fig. 1 correspond to the case of zero spatial angular momentum $J$ : each is the result of averagings over more than 50 trajectories and over long enough integration times [25] to allow for equilibration. This procedure is to be considered a simulation of a microcanonical ensemble [8]. Such an average of the kinetic energy $T$ is denoted as $\langle T\rangle$ and its dependence on the total energy is usually referred to as the caloric curve [26].

The left panels refer to $\mathrm{Ar}_{3}$. Such a cluster already at a relatively low energy exhibits chaotic behavior, the transition to chaos being measured from a growth of the Lyapunov exponent (an index of sensitivity of separation of trajectories for variation of the initial conditions, see Refs. [1-3] and references therein). On the other hand, a possible phase transition to be pinpointed by a growth of the Lindemann factor (a configuration space measure of fluctuations in the interatomic distances, see Ref. [7] and references therein) is poorly observable for such a small system. In clusters of larger sizes, the Lindemann factor is often taken as a signature for occurrence of phase transitions. Some other indicators of chaos and phase transitions in clusters have also been developed in the literature ([1,2,4-6,9] and references therein). The $\mathrm{Ar}_{3}$ system has a global minimum at $-3 \epsilon$ (in the reduced units of Fig. 1 [25]) corresponding to an equilateral triangle, while a
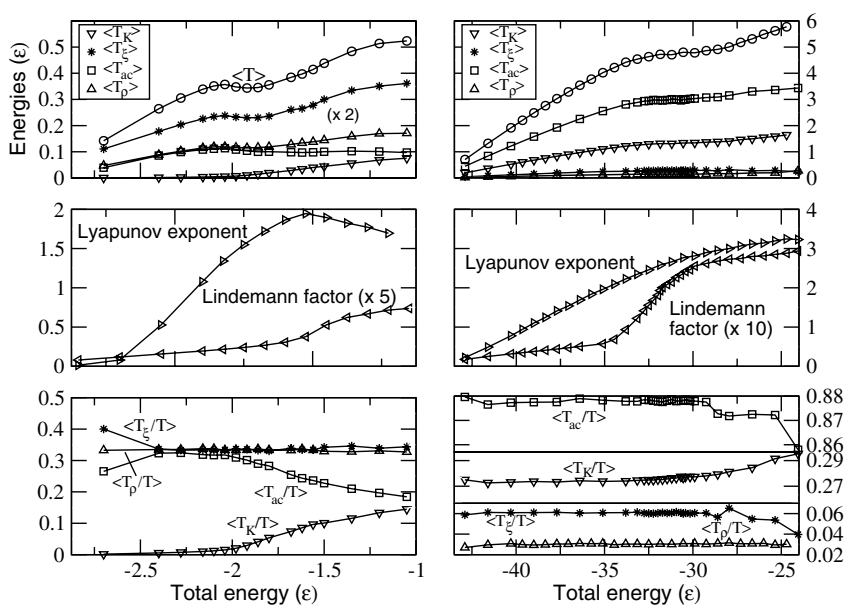

FIG. 1. Molecular dynamics simulations of clusters of three atoms (left panels) and of 13 atoms (right panels), with a Lennard-Jones $(12,6)$ pairwise interaction for $J=0$. The upper panels show the "caloric curve" $\langle T\rangle$ (the microcanonically averaged kinetic energy) and the terms of our partition $\left\langle T_{K}\right\rangle$, $\left\langle T_{\xi}\right\rangle,\left\langle T_{\mathrm{ac}}\right\rangle$, and $\left\langle T_{\rho}\right\rangle$ as functions of the total energy. The middle panels show the dependence on the total energy for two characteristic indicators of classical cluster dynamics, the Lyapunov exponent [1-3] and the Lindemann factor [7]. The lower panels amplify features already apparent in the upper panels, reporting microcanonical averages of the ratios of the various contributions. saddle corresponding to a collinear configuration occurs at $-2.031 \epsilon$. A decrease in the growth of the caloric curve, caused by an opening of the role of an additional path to sample regions of the configuration space by transversing the linear saddle, accordingly shows up in this energy range. For an extensive treatment of chaos in $\mathrm{Ar}_{3}$, see Refs. $[1,2]$.

The upper left panel shows that for this small cluster of atoms, most of the contribution to the caloric curve comes from the deformation modes and that the role of the kinematic rotations is modest, at least at low energies. Very interesting is the observation of how these roles vary with the energy: when the caloric curve decelerates its growth, both the Lyapunov exponent and the Lindemann factor provide signature of onset of chaos and of phase transitions respectively: a rise of the contribution from the kinematic rotations shows up at the expense (at least in relative terms) of their coupling with the deformation modes.

The same type of results is presented in the right panels of Fig. 1, for the case of a 13-atom Lennard-Jones cluster, which is found to exhibit - from the viewpoint of the picture emerging from this study - the behavior paradigmatic of a cluster of moderate size. For such clusters, there is a phase transition clearly apparent in a rise of the Lindemann factor in correspondence of a slowing down in the growth of the caloric curve. This feature is well documented for $\mathrm{Ar}_{13}$ [5]. Our analysis adds insight to the picture. Prominent is a decrease in the relative role of the hyperradial breathing mode $\left\langle T_{\rho}\right\rangle$ : this appears to be typical of all systems at even moderate complexity (this feature may change at higher energies where channels open up for drastic rearrangements or even break-downs). Again a rise of the contribution of the kinematic rotations, which are progressively decoupled from the deformation modes, has to be noted. It can be magnified by considering derivatives of the caloric curve, which would be microcanonical analogues of the heat capacities for canonical simulations.

We have conducted a systematic exploration of the applicability of these tools, which we believe significant to extract physically relevant features hidden in molecular dynamics simulations, to clusters of different sizes and nature, also possessing nonzero spatial angular momentum $J$. These additional studies have confirmed the trends already apparent in Fig. 1. Comparison of the left and right panels yields an exemplification of the fact that, regarding relative roles of the terms of the partition, $\left\langle T_{K}\right\rangle$ increases with the number $N$ of particles in harmony with larger dimension of the kinematic space where the vector $\mathbf{K}$ acts. Further documented is the effect of a growth in the spatial angular momentum $J$, which is to shift all features to higher energies due to an increase of centrifugal barriers.

In this Letter, we have shown in detail how a partition of the kinetic energy of an $N$-body classical system can be 
found upon the introduction of hyperangular momentum tensors. The kinetic energy has been partitioned into five contributions $T_{\rho}$ [Eq. (1)], $T_{J}, T_{K}, T_{\xi}$ [Eq. (2)], and $T_{\mathrm{ac}}$ corresponding to different modes of the system. All the contributions can be calculated from ingredients commonly available in classical trajectory simulations by building up the hyperangular momentum tensors such as $\Lambda, \mathbf{J}, \mathbf{K}$, and $\mathbf{L}_{\xi}$ and by summing the squares of the judiciously chosen elements. Examples of the partition of the kinetic energy for trajectories for $\mathrm{Ar}_{3}$ and $\mathrm{Ar}_{13}$ clusters have been discussed in the paper and shown to point at correlations between the features of the partition and the signatures of chaotic behavior and phase transitions. Other new partitions are being classified [10] in terms of phase-space instantaneous invariants, an ultimate goal being the extraction of those which remain adiabatically invariant [27] under given conditions of practical interest both in the dynamics of nanoaggregates and also in general for reactive systems [28]. This would contribute to elucidating the underlying physics and possibly to suggesting propensities to be exploited as approximate conservation laws, also to simplify the formidable task of the quantum mechanical extension.

We thank Simonetta Cavalli and Andrea Beddoni for useful discussions. We acknowledge the support from the EU through the COST D9 Action and the Human Potential Research Network Theoretical Studies of Electronic and Molecular Processes in Molecules and Clusters (Contract No. HPRN-CT99-00005). This work has also been financed by the Italian MIUR, ENEA, ASI, and by the Turkish Academy of Sciences. M. B. S. gratefully acknowledges the support received from the Italian Ministry of Foreign Affairs organized by the Landau Network - Centro Volta.

*On leave from: Institute of Energy Problems of Chemical Physics, The Russia Academy of Sciences, Leninskir prospect 38, Building 2, Moscow 119334, Russia

[1] R. J. Hinde, R. S. Berry, and D. J. Wales, J. Chem. Phys. 96, 1376 (1992).

[2] R. J. Hinde and R. S. Berry, J. Chem. Phys. 99, 2942 (1993).

[3] E. Yurtsever, Europhys. Lett. 37, 91 (1997).

[4] T. Komatsuzaki and R. S. Berry, Phys. Chem. Chem. Phys. 1, 1387 (1999).

[5] J. Jellinek, T. L. Beck, and R. S. Berry, J. Chem. Phys. 84, 2783 (1986)

[6] D. J. Wales and R. S. Berry, J. Chem. Phys. 92, 4283 (1990).

[7] A. Tekin and M. Yurtsever, Turk. J. chem 26, 627 (2002).

[8] M. Schmidt and H. Haberland, C.r. physique 3, 327 (2002).
[9] R. S. Berry, Nature (London) 393, 212 (1998).

[10] For details of the theory and the definitions of the terms in kinetic energy partitions, see V. Aquilanti, A. Lombardi, and M. B. Sevryuk, J. Chem. Phys. (to be published), available at http://bong.chm.unipg.it/CVs/ aquila.html/Partitions/theory/.

[11] R. G. Littlejohn, K. A. Mitchell, and V. Aquilanti, Phys. Chem. Chem. Phys. 1, 1259 (1999).

[12] V. Aquilanti, A. Beddoni, S. Cavalli, A. Lombardi, and R. G. Littlejohn, Mol. Phys. 98, 1763 (2000).

[13] V. Aquilanti, A. Lombardi, and E. Yurtsever, Phys. Chem. Chem. Phys. 4, 5040 (2002).

[14] F.T. Smith, Phys. Rev. 120, 1058 (1960).

[15] F. T. Smith, J. Chem. Phys. 31, 1352 (1959).

[16] V. Aquilanti and S. Cavalli, J. Chem. Phys. 85, 1355 (1986).

[17] V. Aquilanti, S. Cavalli, and G. Grossi, J. Chem. Phys. 85, 1362 (1986).

[18] V. Aquilanti, L. Bonnet, and S. Cavalli, Mol. Phys. 89, 1 (1996).

[19] R. G. Littlejohn and M. Reinsch, Phys. Rev. A 52, 2035 (1995).

[20] K. A. Mitchell and R. G. Littlejohn, J. Phys. A 33, 1395 (2000).

[21] V. Aquilanti, A. Beddoni, A. Lombardi, and R. G. Littlejohn, Int. J. Quantum Chem. 89, 277 (2002).

[22] V. Aquilanti, S. Cavalli, G. Grossi, and R.W. Anderson, J. Chem. Soc., Faraday Trans. 86, 1681 (1990).

[23] V. Aquilanti, G. Capecchi, and S. Cavalli, Adv. Quantum Chem. 36, 341 (2000).

[24] The terms of the previous single trajectory partition for $T_{\Lambda}$ [13] are not strictly phase-space invariants in the sense proposed here.

[25] We adopt as units the well depth $\epsilon$ of the pair LennardJones potential for energies and the zero location $\sigma$ of this potential for distances. The time-step size in calculations is approximately $2 \times 10^{-14} \mathrm{~s}$. The time scale can be obtained by substitution of the typical values of $\sigma, \epsilon$ and of the argon atom mass $\left(\sigma=3.4 \times 10^{-10} \mathrm{~m}, \epsilon=\right.$ $1.67 \times 10^{-21} \mathrm{~J}$, and $m=39.948$ a.m.u.).

[26] The trajectories computed for generating averaged quantities are obtained by solving the classical Hamilton equations of motion, by a constant time-step four-point Runge-Kutta method. The initial relative positions of the atoms correspond to the minimum energy structure for the system and the energy is given randomly to each degree of freedom.

[27] W. P. Reinhardt, Prog. Theor. Phys. Suppl. 116, 179 (1994); See also S. Wiggins, L. Wiesenfeld, C. Jaffé and T. Uzer, Phys. Rev. Lett. 86, 5478 (2001), where importance of phase-space perspectives in TransitionState-Theory approaches to the dynamics was recently advanced.

[28] See V. Aquilanti, E. Carmona Novillo, E. Garcia, A. Lombardi, M. B. Sevryuk, E. Yurtsever, Comput. Mater. Sci., (to be published). 